
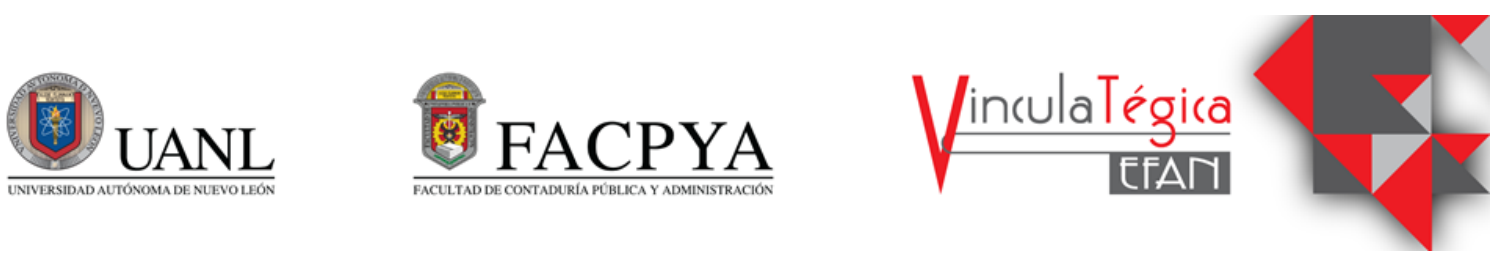

\title{
Instituciones de Educación Superior en la formación de capacidades para el desarrollo
}

\author{
Verónica Loera Suárez ${ }^{1}$, Nidia López Lira² y José Martín Fonseca Moreno ${ }^{3}$ \\ ${ }^{1}$ Universidad Autónoma del Estado de México, Centro Universitario UAEM Valle de Chalco Valle de Chalco, \\ Estado de México, investigacionvls@hotmail.com, Av. Hermenegildo Galeana No. 3, Col. María Isabel Valle \\ de Chalco Solidaridad, (+55)5971 4940. \\ ${ }^{2}$ Universidad Autónoma del Estado de México, Centro Universitario UAEM Valle de Chalco Valle de Chalco, \\ Estado de México, investigacionvls@hotmail.com, Av. Hermenegildo Galeana No.3, Col. María Isabel Valle \\ de Chalco Solidaridad, (+55)5971 4940. \\ ${ }^{3}$ Universidad Mexiquense del Bicentenario, Unidad de Estudios Superiores Chalco Chalco, Estado de \\ México, investigacionvls@hotmail.com, Carretera Federal 115 México-Cuautla s/n Poblado la Candelaria \\ Tlapala Chalco, (+55)59823597.
}

Información del artículo revisado por pares

Fecha de aceptación: junio-2021

Fecha de publicación en línea: diciembre-2021

DOI: https://doi.org/10.29105/vtga7.1-141

\section{Resumen}

En un entorno de cambios constantes y vertiginosos, las Instituciones de Educación Superior (IES) deben diseñar estrategias que les permitan enfrentar ese contexto inestable a nivel global, sobre todo porque en ellas recae la responsabilidad de formar profesionistas cuyo perfil de egreso y aún más, su incorporación al ámbito laboral, favorezca la prevención y solución de problemas sociales, por lo que se requiere de la evaluación de los planes y programas de estudio, para medir su efectividad y tomar decisiones oportunas respecto a la reestructuración de los mismos. La educación es determinante en el desarrollo de cualquier país, por lo que las IES, buscan la formación de profesionistas acordes a las necesidades del entorno, pero, ¿Cuáles son las necesidades de los estudiantes? ¿Qué requieren los empleadores? ¿Qué capacidades favorecen el desarrollo personal y colectivo?, ¿Qué capacidades se están fomentando en los espacios educativos?, estas son algunas interrogantes que dieron origen a la presente investigación, cuyo objetivo fue analizar elementos teóricos sobre el enfoque de formación de capacidades para el desarrollo en instituciones de educación superior en México. El trabajo contempló una investigación documental, misma que permitió examinar la
In an environment of constant and dizzying changes, Higher Education Institutions (IES) must design strategies that allow them to face this unstable context at a global level, especially since they have the responsibility of training professionals whose graduation profile and even more, Their incorporation into the workplace, favors the prevention and solution of social problems, which is why the evaluation of study plans and programs is required, to measure their effectiveness and make timely decisions regarding their restructuring. Education is decisive in the development of any country, so the IES seek the training of professionals according to the needs of the environment, but what are the needs of the students? What do employers require? What capacities favor personal and collective development? What capacities are being promoted in educational spaces? These are some questions that gave rise to this research, whose objective was to analyze theoretical elements on the approach to training capacities for the development in higher education institutions in Mexico. The work contemplated a documentary research, which allowed us to examine the available literature on the role of HEIs in the formation of capacities for development, with the purpose that said information motivates the reflection of the actors in the educational field 
literatura disponible sobre el rol que tienen las IES en la formación de capacidades para el desarrollo, con la finalidad de que dicha información motive la reflexión de los actores en el ámbito educativo (institución, autoridades, docentes y estudiantes) respecto a la relevancia que tiene su participación para lograr el desarrollo individual y colectivo.

Palabras clave: Capacidades, Desarrollo, Formación, IES

\section{INTRODUCCIÓN}

El individuo es un ser social por naturaleza, es por ello que se le contempla como un ser biopsicosocial, lo que significa que cuenta con una serie de características y necesidades biológicas, psicológicas y sociales, para lograr su desarrollo de forma integral.

En el proceso de formación académica formal, las Instituciones de Educación Superior tienen un papel determinante, toda vez que a través de los planes y programas de estudio que ofertan, se contribuye a que los jóvenes adquieran conocimientos y habilidades y consoliden actitudes y valores que les permitan ejercer una profesional de forma eficiente, representando un área de oportunidad para su desarrollo personal y para el desarrollo del contexto en el que se desempeñan.

La presente investigación surge como una de las primeras actividades de un proyecto de investigación a través de la cual se busca analizar cuáles son las capacidades que las Instituciones de Educación Superior en México quieren formar en sus estudiantes, con la finalidad de dimensionar hasta donde atienden las necesidades de desarrollo de los jóvenes y del país, mediante los contenidos educativos de los planes y programas de estudio.

\section{MARCO TEÓRICO.}

Las Instituciones de Educación Superior enfrentan el reto de adaptarse al cambio y rediseñar las estrategias que contribuyan a la formación integral de los jóvenes, de acuerdo a las demandas de la sociedad.

La globalización tiene alcance a (institution, authorities, teachers and students) regarding the relevance of their participation to achieve individual and collective development.

Key words: Capacities, Development, Training, IES. JEL: I21, I23, I31

varios ámbitos, entre los que se pueden mencionar el económico, cultural, social y también el educativo, aspectos que aunados a situaciones como la pandemia del covid 19, han incrementado la necesidad de hacer una análisis respecto a la estructura de los planes y programas de estudio de los diferentes esquemas educativos que ofertan las instituciones de educación superior en el mundo, con la finalidad de evaluar su pertinencia, conocer si son acordes a las exigencias y necesidades del entorno. Para ello es necesario dar respuesta a preguntas como: ¿Las IES cuentan con los elementos necesarios para adaptarse responder a las necesidades del entorno? ¿El personal docente tiene las habilidades adecuadas para transitar de un modelo de educación presencial a un modelo hibrido? ¿La comunidad estudiantil dispone de los elementos necesarios para aprovechar los nuevos modelos de formación académica? ¿Las IES contribuyen a la formación de profesionistas de forma integral? ¿Se está formando a los estudiantes de educación superior con las capacidades necesarias para fomentar el desarrollo? ¿El perfil de los egresados contribuye a resolver problemas sociales?, etc., para responder estas preguntas, se requiere de una investigación profunda en la que se parte del análisis de elementos teóricos para posteriormente realizar investigación de campo que permita conocer la opinión de los sujetos del acto educativo (docentes y estudiantes), así como de los empleadores, egresados y la sociedad.

A continuación se presentan algunos elementos teóricos del tema:

\subsection{Desarrollo}


Esta palabra hace referencia al crecimiento, progreso o evolución positiva de algo, por lo tanto tiene alcance a una actividad, una tarea, una persona, sociedad, región o país. De acuerdo al diccionario de la Real Academia Española, el desarrollo es una acción o efecto de desarrollar o desarrollarse, mientras que desde el punto de vista económico, el término hace referencia a la evolución de una economía hacia mejores niveles de vida.

\subsection{Características del Desarrollo}

Este término puede asociarse a varias áreas de la naturaleza y contexto del ser humano, a continuación se presentan algunas de sus características generales:

$\checkmark$ El desarrollo es universal, lo que significa que tiene alcance a elementos físicos e intelectuales, individuales o colectivos.

$\checkmark$ Se puede generar en forma de proceso, lo que significa que se puede repetir varias veces y que existe interdependencia entre sus etapas.

$\checkmark$ Se asocia como algo positivo, que representa una mejora, crecimiento o aumento de algo.

$\checkmark$ Se lleva a cabo por secciones, que implican cambios o modificaciones en relación a un estado o situación anterior.

\subsection{Tipos de Desarrollo}

La definición de este término, permite identificar el alcance que tiene, en el que se contempla desde un aspecto biológico, hasta el cognoscitivo, de forma individual y colectiva, por lo que de acuerdo a la naturaleza de la presente investigación, se observaran los siguientes tipos:

\subsubsection{Desarrollo cognitivo}

Es el que se lleva a cabo en el individuo y se asocia al proceso a través del cual una persona adquiere, refuerza o consolida conocimientos y desarrolla habilidades, por ejemplo: Aprender cosas nuevas, hablar otro idioma, tener la capacidad de recordar conceptos, lugares, fechas, etc., información que puede y debe poner en práctica, con la finalidad de aprovechar para prevenir o resolver situaciones problemáticas, así como mejorar diversas condiciones.

\subsubsection{Desarrollo personal}

Este tipo de desarrollo se asocia con todas aquellas prácticas que le permiten al individuo satisfacer sus necesidades y experimentar satisfacción personal.

El desarrollo personal está estrechamente ligado con el desarrollo de habilidades blandas, entre las que se pueden mencionar: el autoconocimiento, autocontrol y autoestima, lo que le permite al individuo reconocer sus emociones y sentimientos, además de manejarlos de forma que le permitan superar las áreas de oportunidad personal y relacionarse de mejor manera con otras personas.

\subsubsection{Desarrollo organizacional}

Se logra con la participación de las personas que colaboran en la organización, toda vez que se considera al capital humano como el activo más importante de la entidad, porque a través de sus conocimientos, habilidades y actitudes, se favorece el logro de las metas y objetivos de la empresa o institución; por lo que se recomienda fomentar una cultura en la que los objetivos personales de los colaboradores se alineen con los objetivos organizacionales, lo que beneficiará el desarrollo organizacional.

\subsubsection{Desarrollo económico}

Este tipo de desarrollo se asocia con la capacidad que tiene un país o región, de generar riqueza para sus habitantes, considerando todos los recursos disponibles entre los que se pueden mencionar los naturales, financieros, tecnológicos e incluso los conocimientos y habilidades de su población, lo que permitirá el 
aprovechamiento del resto de los recursos. A través del este tipo de desarrollo, se generan las condiciones que favorecen la satisfacción de las necesidades básicas de una población en áreas de alimentación, salud, vivienda, educación, servicios, etc.; elementos clave para generar condiciones que contribuyan a la calidad de vida de los habitantes, es por ello, que a nivel mundial, los diferentes gobiernos tienen como un objetivo principal, el desarrollo económico del país, estado o región

\subsubsection{Desarrollo humano}

Este tipo de desarrollo se logra, cuando se tiene una percepción colectiva de que se cuenta con los elementos necesarios para satisfacer las necesidades de un individuo y su familia, aspectos que se traducen en calidad de vida para la población; para esto se requiere la participación y aportación de los miembros de una sociedad, toda vez que el ser humano es un ser social por naturaleza, lo que significa que para satisfacer sus necesidades, requiere de la participación de otras personas, a través de la oferta de bienes y servicios y de la misma interacción social. En este tipo de desarrollo el objetivo es alcanzar el bienestar de la población de manera colectiva.

De acuerdo a los diferentes tipos de desarrollo, se puede observar que están estrechamente ligados y que en muchos de los casos, para lograr un desarrollo especifico, se requiere de otro previo, por ejemplo, para lograr el desarrollo humano, primero se debe alcanzar el desarrollo económico.

\subsubsection{Desarrollo social}

Se alcanza este nivel de desarrollo, cuando se mejoran las condiciones de vida de forma colectiva, lo que representa un proceso de evolución para un país o región, en donde las mejoras y beneficios tengan un mayor alcance, por lo que se puede decir, que para lograr este nivel de desarrollo, ya debieron alcanzarse los diferentes niveles de desarrollo mencionados previamente.

El desarrollo social tiene que ver con un crecimiento económico, a través del cual, las personas tengan acceso empleos dignos y bien remunerados, que les permitan adquirir alimentos para ellos y sus familias, vivienda digna, servicios de salud de calidad, educación adecuada en los diferentes niveles, lo que significa igualdad de oportunidades y equidad social.

Después de revisar algunos conceptos de los diferentes tipos de desarrollo, a continuación se presentan algunas definiciones de capacidades.

\subsection{Capacidades}

De acuerdo a González (2021), es difícil construir una definición única e integral de esta palabra, toda vez que es considerado un término ambiguo y solo se puede tener una aproximación de su significado, por lo tanto, la capacidad suele referirse a conocimiento, aptitud, potencial y/o habilidad.

Rueda (2014), menciona que el desarrollo de capacidades consiste en mejorar las condiciones en las que se desenvuelvan las personas, contemplando aspectos desde lo individual a lo colectivo, por ejemplo: el aprender algo nuevo, el fortalecer una habilidad, lo que representaría un beneficio para la persona, mismo que se puede hacer extensivo a otros individuos.

De acuerdo a Amartya Sen, citado por Rueda (2014), el desarrollo de capacidades se asocia a fomentar en el ser humano, la adquisición y consolidación de conocimientos, habilidades y actitudes, mismas que debe poner en práctica buscando el beneficio personal y colectivo, para lo que se requiere una participación más activa, que convierta a los individuos en motores de cambio.

Para Gómez y de Alba, citados por González (2021), la capacidad es algo intangible, pero de gran relevancia, toda vez que representa el medio a través del cual las personas tienen un desempeño que permite alcanzar resultados eficientes de acuerdo a las necesidades $\mathrm{e}$ intereses individuales $\mathrm{y}$ colectivos. Los autores mencionan que de forma general, las capacidades representan la habilidad para reflexionar y plantear metas y 
objetivos, diseñar estrategias y ponerlas en práctica para lograrlos y resolver problemas de forma sostenible.

Para el Programa de Naciones Unidas para el Desarrollo (PNUD), el desarrollo de capacidades es el proceso mediante el cual las personas, organizaciones y sociedades, obtienen, fortalecen y mantienen las aptitudes necesarias para establecer y alcanzar sus propios objetivos de desarrollo a lo largo del tiempo.

Henríquez (2013), menciona que el enfoque de las capacidades está estrechamente ligado con naturaleza del individuo y la relación que se da entre él y los recursos disponibles que le permiten alcanzar sus objetivos y satisfacer sus necesidades, favoreciendo su calidad de vida. El autor hace una cita de Sen (2005), mencionando que las personas son física y mentalmente heterogéneas, viven en medios diversos y tienen acceso dispar a bienes y servicios, provocando que sus necesidades sean también variadas y en consecuencia, el desarrollo de capacidades dependerá del contexto del individuo y los recursos a los que tenga acceso.

Para Sanz y Serrano (2016), el desarrollo de capacidades en la educación, es una cuestión de justicia social, toda vez que el derecho a la educación es un derecho humano al que todos los individuos deben tener acceso; las instituciones educativas deben ofrecer educación de calidad que permita la igualdad de condiciones entre sus estudiantes. De acuerdo a estos autores, la educación además de favorecer el desarrollo de capacidades, contribuye a que los individuos conozcan y reconozcan sus derechos.

De acuerdo a los elementos teóricos previamente mencionados, las capacidades pueden ser interpretadas como herramientas que le permiten a los individuos, desarrollar actividades en ámbitos personales, académicos, laborales y sociales, es por ello que las IES tienen un papel determinante en la formación de profesionistas, toda vez que no basta con que adquieran conocimientos de las disciplinas en las que se estén formando, además del aspecto cognoscitivo, se requiere que desarrollen habilidades y actitudes que les permitan incorporarse al ámbito laboral de forma efectiva, siendo motores de cambio que contribuyan a la prevención de problemas y la solución de los mismos.

\subsubsection{Características de las capacidades}

Diversos autores han realizado análisis que permiten reflexionar sobre las peculiaridades de las capacidades, tal es el caso de Martínez, citado por González (2021), quien expone que las principales características de las capacidades son:

1) Son dinámicas, cambian, se modifican con frecuencia;

2) Se logran a través de la combinación de conocimientos, habilidades y recursos;

3) Se pueden enriquecer o fortalecer, pero también se pueden debilitar y en consecuencia, eliminar;

4) Son específicas, dependiendo de las personas y su contexto;

5) Requieren de mantenimiento permanente, $\mathrm{y}$

6) Se ponen en práctica de forma diversa, de acuerdo a la situación, las personas y los recursos disponibles.

De acuerdo a lo antes expuesto, se puede reflexionar sobre los factores que inciden en el desarrollo y consolidación de capacidades, mismas que no se pueden generalizar toda vez que dependerá del individuo, los recursos disponibles, las capacidades previas de las que disponga para la construcción de nuevas y la interacción que tenga con otros individuos y la naturaleza de las capacidades con las que cuenten.

Otro elemento importante por resaltar, es el que una vez desarrolladas las capacidades, no son permanentes, su consolidación dependerá de la frecuencia con la que se practiquen y la utilidad que representen, provocando que puedan llegar a debilitarse e incluso, destruirse, al dejar de representar un beneficio para el individuo.

La eficiencia de las mismas, 
dependerá de los actores y el contexto en el que fueron desarrolladas y practicadas, así como su efectividad para prevenir o resolver problemas específicos.

\subsubsection{Enfoque de las capacidades}

De acuerdo a Gasper, citado por Dubois (2008), una de las críticas al enfoque de las capacidades, es la concepción individualista con la que se aborda el tema, al considerar que depende de los recursos y capacidades previas de los individuos, cuyo objetivo es lograr el bienestar individual, pero para lograr el desarrollo humano, es indispensable partir del desarrollo de capacidades individuales con un impacto colectivo, solo de esa forma se puede construir el escenario en el que la sociedad puede tener acceso a condiciones que favorezcan la calidad de vida de las personas.

\subsection{Competencias}

De acuerdo a Bustamante, citado por Cejas, Rueda et al. (2019), la palabra competencia es asumida como un concepto "recontextualizado que se ha transformando, con base a los significados que originalmente se han proporcionado en diferentes contextos", sin embargo, el concepto ha sido entendido como aquel que tiene muchas dimensiones, determinándose su significación desde los diversos ámbitos tales como el educativo, laboral, cultural, social y más aún, en la gestión humana.

Para la Organización Internacional del Trabajo (OIT), una competencia es una síntesis de capacidades volitivas, procedimentales y cognitivas.

La formación con base en competencias, contempla los siguientes tres dominios: cognoscitivo, lo afectivo y lo psicomotor.

De acuerdo a estos dominios, se requiere que el individuo no solo tenga conocimientos, sino que los ponga en práctica $\mathrm{y}$ que lo haga de forma armoniosa al interactuar con otras personas.

El informe de Delors, "La educación encierra un tesoro" (1996), hay cuatro pilares en la educación que tienen que ver con la formación:

1) Aprender a conocer: Dominar los instrumentos del conocimiento, vivir dignamente y hacer su propio aporte a la sociedad.

2) Aprender a hacer: Lo cual significa que se debe aprender para hacer cosas y se debe preparar al futuro profesional, conocimientos que agregan valor a la sociedad.

3) Aprender a convivir y a trabajar en proyectos comunes: Esto implica que no será posible comprender las acciones de uno mismo sino se aprende a conocer al otro, para ello hay que intentar romper con la diversidad y crear más igualdad.

4) Aprender a ser: Es el desarrollo total y máximo posible de cada persona. (Cejas, Rueda et al. 2019).

\subsection{Formación integral}

De acuerdo a Nova (2017), el hombre por su naturaleza requiere de un proceso de formación para su crecimiento personal, intelectual y social.

La autora menciona que para lograr una formación integral, se requiere de una formación humana. De acuerdo al filósofo Orozco (1999), citado por Nova (2017), las Instituciones de Educación Superior, tienen una responsabilidad importante para fortalecer la formación integral de sus estudiantes, toda vez que en este espacio se consolida el carácter, la personalidad, la ética y el pensamiento crítico.

Delors (1996) amplia esta visión, al describir la misión de la universidad, centrándose en el impacto que tienen a nivel social. El autor menciona que en esta etapa de formación, los estudiantes deben adquirir conocimientos en las diferentes disciplinas, pero también se deben consolidar los valores universales; concibe el rol de la universidad de forma integral, formando personas en las cuatro áreas sustantivas que son la base de las instituciones educativas en el nivel superior, las cuales son: docencia, investigación, cultura y extensión y vinculación, a través de las 
cuales se enriquezca el perfil de egreso de los profesionistas, lo que se verá reflejado en una participación activa para prevenir y resolver problemas de su entorno.

De acuerdo a Misas (2004), citado por Nova (2017), las instituciones de educación superior deben formar profesionales que se comprometan con la construcción de una nueva sociedad. De esta manera, se aportará al cimiento del desarrollo económico, social y político. Para tal fin, dentro de los propósitos de los centros universitarios estará: aumentar el conocimiento por medio de la investigación, transmitir saberes y valores dentro de un clima social y cultural, y la formación ética-estéticacientífica, la cual permite al individuo desarrollar sus potencialidades y capacidades imaginativas. Esto quiere decir que la ciencia, la tecnología, los conocimientos, las habilidades y destrezas físicas, artísticas, la ética, y demás aspectos que aporten al crecimiento humano, social e intelectual, deberán ser tratados con la misma importancia.

\subsection{Rol de las IES en la formación de capacidades para el Desarrollo}

Las Instituciones de Educación Superior, enfrentan el reto de lograr la formación integral de sus estudiantes en las diferentes disciplinas en las que se forman, se contempla como un reto, porque se debe contar con evidencia del objetivo logrado, la cual debe ir mas allá de lo mencionado en los planes y programas de estudio, se debe contar con opiniones favorables de los empleadores, los egresados y la sociedad; es por ello, que se deben diseñar instrumentos que contribuyan a una investigación que permita obtener este tipo de información, misma que también enriquecerá, la actualización de los planes y programas de estudio.

Badillo (2007), en su reseña de los retos de México en el futuro de la educación, presentado por el Consejo de especialistas para la educación, el cual fue creado en el 2005, como una dependencia de apoyo a la Secretaria de Educación Pública en materia de planeación y política educativa, menciona que los integrantes de dicho Consejo hacían referencia a los propósitos generales que debía tener la educación, los cuales fueron: una educación para la justicia y la equidad; una educación para la democracia, la cohesión social y la responsabilidad ciudadana; y una educación para la productividad, la competitividad y el desarrollo, siendo esta la parte medular en ese primer análisis realizado por la dependencia. En el texto se reafirmaba que el propósito fundamental debería ser la formación de las personas, de las nuevas generaciones, la formación de ciudadanos capaces de hacer crecer el país, contribuyendo a su propio bienestar y al de su entorno.

De acuerdo a la Asociación Nacional de Universidades e Instituciones de Educación Superior (ANUIES, 2018), desde el inicio del siglo XXI se ha acelerado la convergencia de las tecnologías digitales, físicas y biológicas, lo que ha permitido nuevos desarrollos en los campos de la inteligencia artificial, la automatización, la comunicación y el trabajo. Estos incesantes avances están modificando las necesidades de aprendizaje y las habilidades cognitivas y socioemocionales que requieren los egresados de la educación superior.

El impulso de un nuevo enfoque en las políticas públicas permitirá transitar de un sistema cerrado de educación superior centrado en las tradicionales formas de operar de las IES a un sistema abierto, flexible, innovador, articulado y dinámico que potencie la contribución de las IES y faciliten la construcción de una mejor sociedad.

Las universidades, los centros de investigación y las instituciones tecnológicas y pedagógicas cuentan con un rico capital intelectual para hacer aportaciones significativas en cada uno de los objetivos de la Agenda que contempla, a partir de una visión de futuro ambiciosa y de cambio sobre las tres dimensiones del desarrollo sostenible —económica, social y ambiental—, un mundo en el que la alfabetización sea universal, con acceso equitativo a una educación de calidad en sus distintos niveles, a fin de que todas las personas, especialmente quienes se encuentran en situación de vulnerabilidad, cuenten con un 
entorno propicio para la plena realización de sus derechos y capacidades y para su participación activa en el desarrollo de la sociedad (ANUIES 2018).

En los próximos años se acelerará la conversión de las Tecnologías de Información y Comunicación (TIC) a Tecnologías para Aprendizaje y el Conocimiento (TAC) en todos los espacios educativos, tanto presenciales, a distancia, híbridos y abiertos; se modificará la concepción rígida y disciplinaria de los programas educativos para dar paso a un aprendizaje flexible en donde existan alternativas en cuanto a cómo, cuándo, dónde y qué estudiar; el estudiante será el centro en los procesos de enseñanzaaprendizaje y el aprendizaje experiencial y activo tendrán un papel clave en la formación de las competencias necesarias para el siglo XXI; la investigación se convertirá cada vez más en el soporte de la formación y las instituciones educativas tendrán una aportación significativa al desarrollo de su entorno.

De acuerdo con Joseph Aoun (2017), para garantizar una educación "a prueba de robots", será necesario que las IES combinen la enseñanza de la ciencia y la tecnología con las ciencias sociales y las humanidades. La educación para el futuro "se concentrará en desarrollar las habilidades y competencias netamente humanas para las cuales, al menos por ahora, las máquinas no ofrecen una alternativa viable, tales como la empatía, el trato personal y las relaciones de grupo.

\section{MÉTODO}

La presente investigación se realizó de forma documental, haciendo un análisis de la literatura sobre los temas de capacidades, competencias, desarrollo y el rol de las Instituciones de Educación Superior en la formación de profesionistas atendiendo las demandas de la sociedad.

El enfoque de la investigación es cualitativo y tiene un alcance descriptivo, toda vez que en esta etapa de la investigación se analizan las aportaciones teóricas de diversos autores para dar respuesta a las preguntas de investigación planteadas.

\section{RESULTADOS}

De acuerdo al análisis de la literatura, se confirma la importancia que tienen las Instituciones de Educación Superior en la formación de profesionistas con un enfoque integral, cuyos conocimientos representen capacidades que favorezcan el desarrollo del individuo y de los diferentes actores del contexto en el que se desempeñe, por ejemplo, su familia, amigos, el ámbito laboral y social.

Las IES enfrentan retos ambiciosos derivados del impacto de la globalización y el contexto inestable en el que se ubica la educación a nivel mundial, así como las necesidades sociales y los problemas organizacionales. Se requiere que las IES oferten programas educativos de calidad y acordes a las nuevas necesidades, en las cuales está presente el aspecto tecnológico para aprovechar los diferentes recursos disponibles y contribuir a que la brecha con la población vulnerable de México, sea más estrecha; sin embargo, aunque se han realizado varios esfuerzos por mejorar esta situación, aún hay un camino importante por recorrer, en donde se requiere que los estudiantes cambian su rol pasivo por uno participativo al adquirir, desarrollar y consolidar diversas capacidades cognoscitivas y sociales, con la finalidad de ponerlas en práctica de forma inmediata, sin tener que esperar a concluir el plan de estudios, bajo estricta responsabilidad, esto es, aplicar los conocimientos adquiridos.

El análisis teórico presentado en el presente trabajo, representa una de las actividades iniciales de una investigación más profunda que tiene la finalidad de identificar las capacidades que las IES en México, buscan fomentar entre sus estudiantes, así como evaluar la pertinencia de acuerdo a los contenidos de los planes y programas de estudio, haciendo un análisis comparativo con las necesidades de los jóvenes universitarios, así como de los empleadores y la sociedad en general, para lo cual se requiere realizar diversas actividades entre las que se 
encuentran:

1) Realizar un análisis de los programas educativos ofertados respecto a los de mayor demanda en México.

2) Revisar la pertinencia de los contenidos de los planes de estudio, considerando la estructura (unidades de aprendizaje que los conforman), así como la aportación que hacen al perfil de egreso.

3) Diseñar instrumentos de recolección de datos que permitan conocer la opinión de los empleadores, egresados y la sociedad en general, respecto a la pertinencia de los planes y programas de estudio.

4) Profundizar el análisis de los retos de la educación en México, con la finalidad de proponer estrategias que favorezcan el logro de los objetivos de ofertar educación de calidad en todos los niveles educativos y de acuerdo a la naturaleza y alcance la investigación, enfocándose en el nivel superior.

También se resalta la importancia que tiene el consolidar un modelo de educación incluyente, en el que se generen alianzas en las que participen los actores de la institución como son directivos, administrativos, docentes y estudiantes y el sector organizacional, incluyendo a la iniciativa pública y privada, toda vez que ambas forman parte del campo laboral de los diferentes programas de estudio.

\section{CONCLUSIONES}

Las Instituciones de Educación Superior deben ofertar programas educativos de calidad que contribuyan a que sus estudiantes $\mathrm{y}$ egresados adquieran, desarrollen y consoliden capacidades orientadas a lograr el desarrollo individual y colectivo, beneficiando al estudiante, egresado y demás personas del contexto en el que se desenvuelva.

Las capacidades deben tener un alcance amplio que contemple el aspecto cognoscitivo, afectivo y social.

Se debe continuar con el enfoque de la formación integral de los estudiantes en las IES de México, toda vez que a través de él, el perfil de egreso se enriquece porque los estudiantes reciben formación disciplinar, pero también en temas sustantivos como son la investigación, la cultura, el deporte, entre otros.

Las IES deben realizar las gestiones necearías que contribuyan a disminuir la brecha con los sectores vulnerables del país que no tienen acceso a educación de nivel superior.

Gestionar recursos para invertir en tecnología que favorezca el acercamiento de la educación a las zonas en las que actualmente no hay oportunidades para recibir educación formal en este nivel.

Las IES deben fomentar un proceso de actualización constante para todos los colaboradores que participan y hacen posible la misión de la universidad.

Se recomienda complementar la investigación con el trabajo de campo para enriquecer los resultados y lograr los objetivos planteados en el proyecto de investigación que dio origen al presente trabajo. 
Vol. 7 Núm. 2 


\section{REFERENCIAS}

ANUIES (2018). Propuesta de la ANUIES para renovar la educación superior en México. Disponible en: https://visionyaccion2030.anuies.mx/Vision_accion2030.pdf

Ascón Villa, Jimmy Eduardo, García González, Máryuri, \& Lajara Cruz, Adrián de Jesús. (2019). Pirámide para el desarrollo de habilidades directivas en las instituciones de educación superior (IES). Revista Cubana de Educación Superior, 38(3), e5. Epub 01 de diciembre de 2019. Disponible en: http://scielo.sld.cu/scielo.php?script=sci_arttext\&pid=S02573142019000300005\&lng=es\&tlng=es.

Badillo Guzmán, Jessica (2007). Reseña de "Los retos de México en el futuro de la educación" de Consejo de Especialistas para la Educación. CPU-e, Revista de Investigación Educativa, (4), 1-11. Disponible en: https://www.redalyc.org/articulo.oa?id=283121710012

Carro Olvera, Adriana y Lima Gutiérrez, José Alfonso (2015). El papel de la escuela localmente relevante frente a las actuales teorías del desarrollo. Un acercamiento al enfoque de las capacidades humanas. Diálogos sobre educación. Temas actuales en investigación educativa, 6 (10), 1-22. Disponible en: https://www.redalyc.org/articulo.oa?id=553457060004

Cejas Martínez, Magda F, \& María José, Rueda Manzano, \& Cayo Lema, Luis Efraín, \& Villa Andrade, Luisa Carolina (2019). Formación por competencias: Reto de la educación superior. Revista de Ciencias Sociales (Ve), XXV (1), ISSN: 1315-9518. Disponible en: https://www.redalyc.org/articulo.oa?id=28059678009

Diccionario de la RAE. Desarrollo. Disponible en: https://dle.rae.es/desarrollo

Dubois Migoya, Alfonso (2008). El debate sobre el enfoque de las capacidades: las capacidades colectivas. Araucaria. Revista Iberoamericana de Filosofía, Política y Humanidades, 10 (20), 35-63. ISSN: 1575-6823. Disponible en: https://www.redalyc.org/articulo.oa?id=28212043003

Arteaga. El desarrollo humano y la generación de capacidades. Disponible en: https://core.ac.uk/download/pdf/47265029.pdf

García-García, Francisco J. (2018). Educación y capacidades. Hacia un nuevo enfoque del desarrollo humano. José Antonio Ibáñez-Martín y Juan Luis Fuentes (coordinadores), Madrid, Dykinson SL, 2017. Perfiles Educativos, XL (162), 206-210. ISSN: 0185-2698. Disponible en: https://www.redalyc.org/articulo.oa?id=13258437014

González Bazaldua, Hugo Alejandro (2021). Capacidades: (otra vez) un análisis conceptual y metodológico. Intersticios Sociales, (21), 9-43. ISSN:. Disponible en: https://www.redalyc.org/articulo.oa?id=421766332002

Hernández (2013). Del contrato social al enfoque de las capacidades. Revista de Derecho Universidad Católica del Norte, 20 (1), 265-291. ISSN: 0717-5345. Disponible en: https://www.redalyc.org/articulo.oa? $\mathbf{i d = 3 7 1 0 4 1 3 2 7 0 1 0}$

Jiménez, Hernández \& González (2013). Competencias profesionales en la educación superior: justificación, evaluación y análisis. Innovación educativa (México, DF), 13(61), 45-65. Disponible en: http://www.scielo.org.mx/scielo.php?script=sci_arttext\&pid=S166526732013000100004\&lng=es\&tlng=es.

Leyva, Ganga, Tejada \& Hernández (2015). La formación por competencias en la educación superior: Alcances y limitaciones desde referentes de México, España y Chile. Disponible en:

http://eprints.uanl.mx/10923/1/Libro\%20Formaci\% C3\%B3n\% 20por\%20Competencias.pdf

Miranda Torrez, Julián (2015). El Modelo de las Capacidades Dinámicas en las Organizaciones. Investigación Administrativa, 44 (116), 81-93. ISSN: 1870-6614. Disponible en: https://www.redalyc.org/articulo.oa?id=456044959005

Nova (2017). Formación integral en la educación superior: análisis de contenido de discursos políticos. Praxis \& Sabre, 8 (17), 181-200. ISSN: 2216-0159. Disponible en: https://www.redalyc.org/articulo.oa?id=477253330009

Programa de la Naciones Unidas para el Desarrollo (PNUD 2009). Desarrollo de capacidades. Disponible en: https://www.undp.org > undp > library > spanish 
Rueda Bouillon, Julian (2014). El desarrollo de capacidades, un enfoque central de la cooperación al desarrollo. Disponible en:

http://diposit.ub.edu/dspace/bitstream/2445/66912/1/Memoria_Julian_Rueda_Bouillon.pdf

Sanz \& Serrano (2016). El desarrollo de capacidades en la educación. Una cuestión de justicia social. Sinéctica, (46). Disponible

http://www.scielo.org.mx/scielo.php?script=sci_arttext\&pid=S1665109X2016000100004\&lng=es\&tlng=es. 